\title{
Inefficient health care costs lives: $\mathrm{CIHI}$
}

Inefficient health care may cost Canada tens of thousands of lives each year, suggests a Canadian Institute for Health Information (CIHI) report.

Health regions could reduce deaths due to treatable causes, such as asthma and sepsis, by $18 \%-35 \%$ by working more efficiently, according to the Apr. 10 report, Measuring the Level and Determinants of Health System Efficiency in Canada. That translates to the potential prevention of 12600 24500 premature deaths per year, at no extra cost.

"We can do more with what we have," says Senior Researcher Sara Allin. "These are deaths that should really be avoided in the presence of timely and effective health care."

Part of the problem may be that traditional efforts to improve efficiency have focused almost exclusively on the management and organization of health services, she adds.

CIHI's analysis of performance variations across 89 health regions reveals a host of other factors at play, including the overall health of a region's population and how easily people from lower-income groups were able to access care.

Researchers found that higher levels of efficiency were "significantly associated" with indicators of the successful prevention and management of chronic disease, such as lower rates of smoking, physical inactivity, obesity, multiple chronic conditions and hospital readmissions.

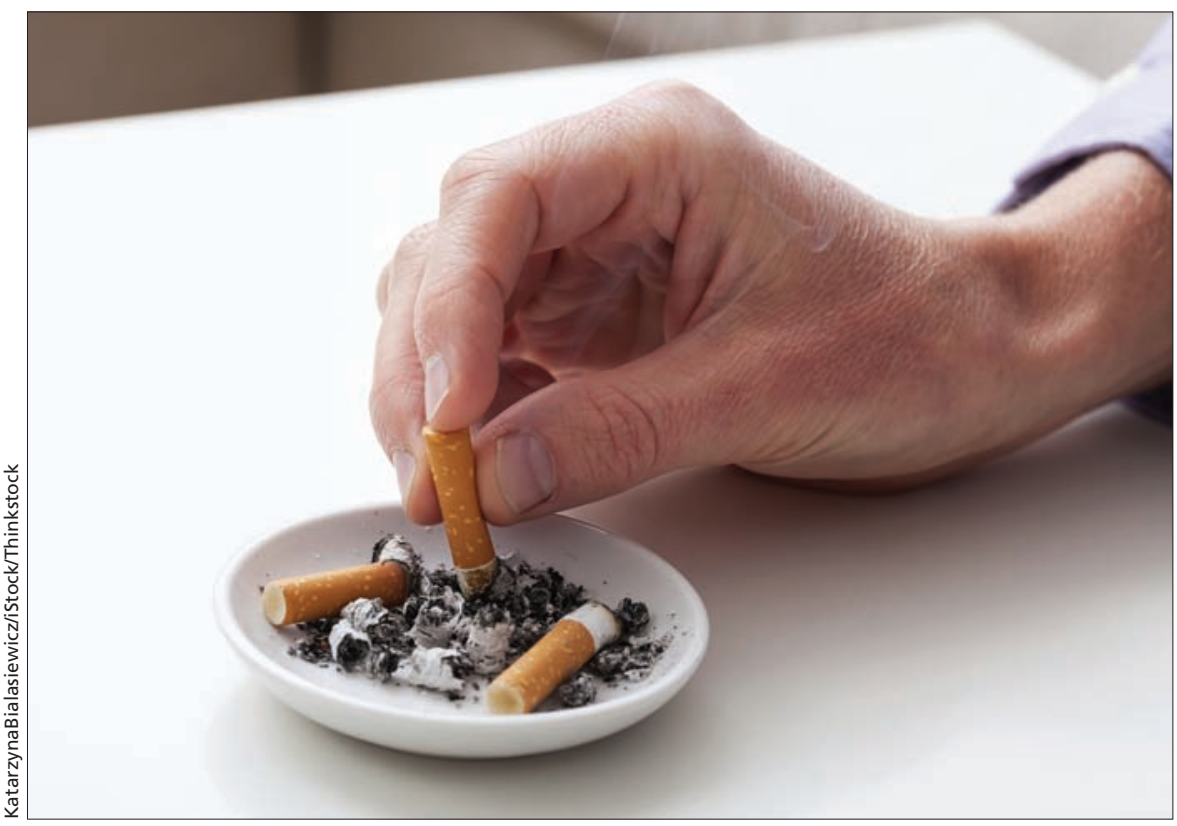

Reducing risk factors, such as smoking, for disease will ensure Canada gets more value for its health dollars, says $\mathrm{CIHI}$.

Notably, "if you could reduce the prevalence of smoking in a region by 10 percentage points, efficiency would increase $10 \%$ as a result," says Allin. Similar reductions in physical inactivity and multiple chronic conditions boosted efficiency by $7 \%$ and $12 \%$, respectively.

Regions that provided better access to care for people from lower-income groups also received better value for their health dollars. Other factors associated with higher levels of efficiency included greater investment in primary care and shorter hospital stays among patients waiting for transfer to nonhos- pital facilities, a group mostly comprised of seniors.

Based on these findings, CIHI is urging health regions to expand their efficiency efforts to focus greater attention on disease prevention and equitable access to care.

"You can only get so far by focusing on the organization and delivery of services," says Allin. "Population interventions are needed to reduce those risk factors that cause people to enter the health system in the first place." Lauren Vogel, CMAJ

CMAJ 2014. DOI:10.1503/cmaj.109-4783 